ИЗВЕСТИЯ АКАДЕМИИ НАУК ЭСТОНСКОП ССР. ФИЗИКА * МАТЕМАТИКА PROCEEDINGS OF THE ACADEMY OF SCIENCES OF THE ESTONIAN SSR. PHYSICS * MATHEMATICS

1986, 35, 2

\title{
ОПРЕДЕЛЕНИЕ ИНДУКТИВНОСТИ РАЗДЕЛИТЕЛЬНЫХ РЕАКТОРОВ В СИСТЕМЕ ЭЛЕКТРОПИТАНИЯ С МАГИСТРАЛЬНОЙ РАСПРЕДЕЛИТЕЛЬНОЙ СЕТЬЮ
}

T. TOMSON. ERALDUSREAKTORITE INDUKTIIVSUSE MAARAMINE MAGISTRAALI TOOPI JAOTUSVORGUGA ELEKTRITOITESUSTEEMIDES

T. TOMSON, INDUCTANCE DETERMINATION OF ISOLATION REACTORS IN POWER SYSTEMS WITH MAIN-TYPE DISTRIBUTION NET

\section{(Представил И. Эпик)}

Применение магистральной распределительной сети в системе электропитания (СЭП) на базе управляемых тирнсторных выпрямителей с общей для них частью [1] весьма желательно. Однако это требует технически сложных длинных управляющих импульсов $\left[{ }^{2}\right]$ или предложенных в $\left.{ }^{3}\right]$ разделительных «анодных» реакторов с индуктивностью рассеивания $L_{a}$, методика расчета которых не приспособлена для случая магистральной сети и уточняется в настоящей статье.

Настоящий анализ, который одинаково правомерен для СЭП как на базе нулевых, так и мостовых выпрямителей, построен с учетом следующих упрощающих предпосылок:

1) тиристоры аппроксимируются идеальными ключами,

2) трансформатор аппроксимируется индуктивностью рассеивания $L_{s}$,

3) сеть аппроксимируется индуктивностью $L_{c}$, все участки сети, как и нагрузки вентильных групп, симметричны,

4) нагрузка аппроксимируется генератором тока $I_{d}$.

Проанализируем наиболее тяжелый случай синфазного управления при угле $\alpha$ для малого числа вентильных групп $n \in\{2,3\}$. Наиболее тяжелые условия коммутации, очевидно, создаются для пары вентилей в конце цепи. Для этой пары дополнительные реакторы не нужны. Схема замещения контура коммутации при $n=3$ показана на рнс. 1 в развернутом виде и на рис. 2 в преобразованном виде. Эквивалентную индуктивность цепи и эквивалентное ЭДС обозначим левее осевой линии (рис. 1) как $L_{\text {сэп }}$ и $e_{\text {сэп }}(p)$ соответственно. Указанные эквивалентные величины могут быть найдены по общеизвестным правилам преобразования линейных электрических цепей. Приведем здесь лишь

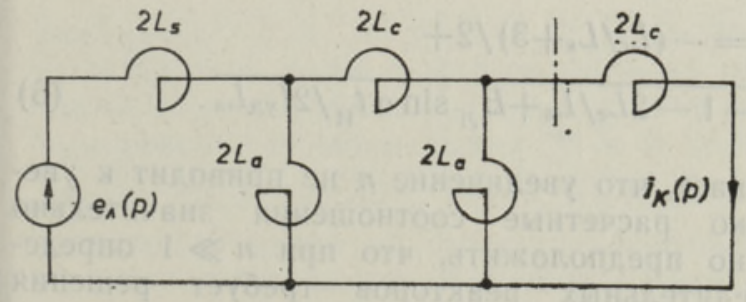

Рнс. 1

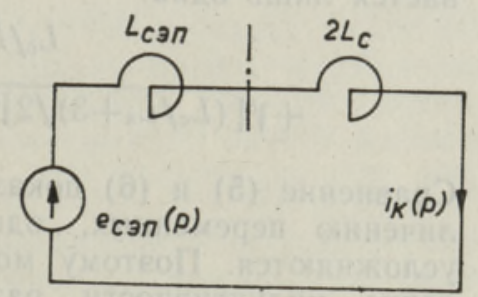

Рис. 2 


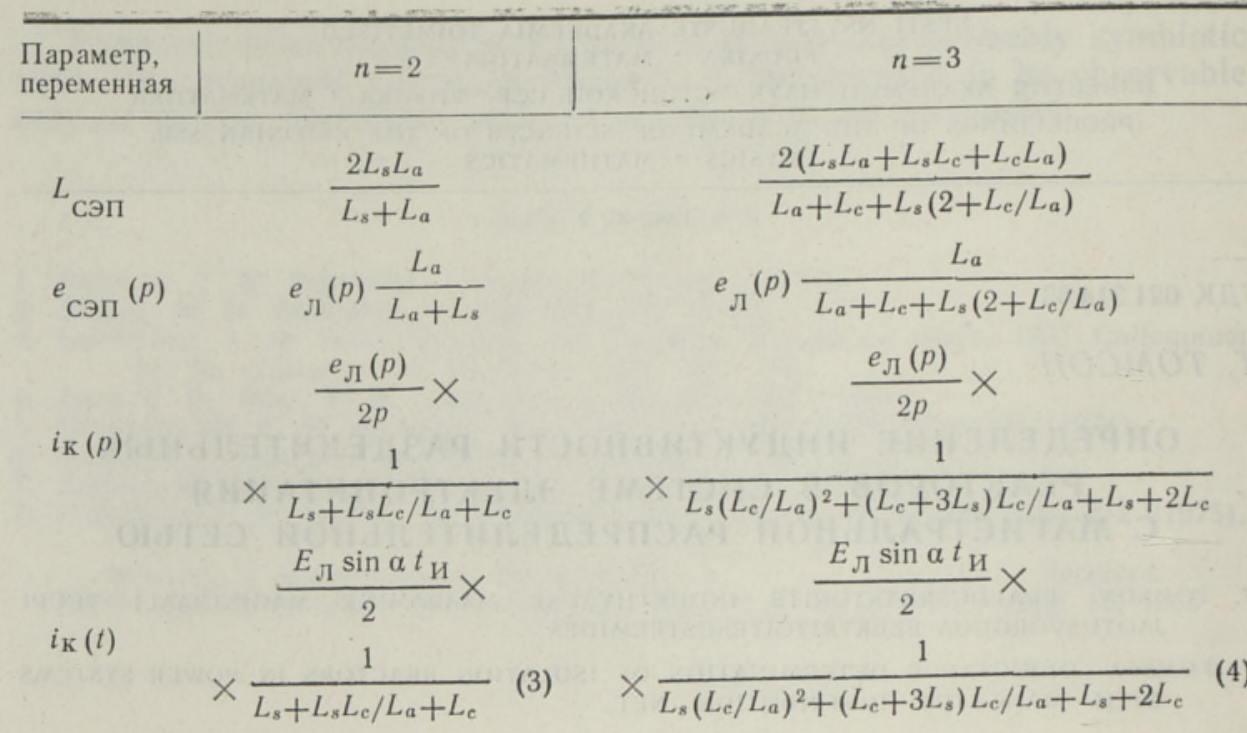

их результирующие значения, указанные в таблице. Ток коммутации $i_{K}(p)$ в конце цепи может быть найден

$$
i_{K}(p)=\frac{e_{\text {сэп }}(p)}{p} \frac{1}{L_{\text {сэп }}+2 L_{c}} .
$$

Условием удачной коммутации является

$$
i_{K}\left(t_{\mathrm{H}}\right) \geqslant I_{\mathrm{yд}},
$$

где $t_{\text {и }}-$ длительность короткого $t_{\text {и }} \rightarrow 0$ управляющего импульса, $I_{\text {уд }}$ - ток удержания тиристора, $\gamma-$ угол коммутации, $\omega-$ частота сети, $E_{\text {л }}$ - амплитуда линейного напряжения вентильной обмотки. Так как нас интересует лишь начало коммутации, то без существенной эшибки можем считать $e_{\text {Л }}(p)=E_{л} \sin \alpha-$ значение, которое ЭДС имеет в момент подачи управляющего импульса. С использованием этого упрощения найдены оригиналы (1), указанные в таблице. По оригиналам (3) и (4) с учетом (2) могут быть получены необходимые расчетные соотношения. Для СЭП при $n=2$

$$
L_{c} / L_{a}=E_{\text {Л }} \sin \alpha t_{\text {И }} / 2 I_{\text {уд }} L_{s}-1-L_{c} / L_{s} .
$$

Для СЭП при $n=3$ выражение $L_{c} / L_{a}$ из (4) приводит к появлению квадратичного управления, из корней которого действительной оказывается лишь одна:

$$
\begin{gathered}
L_{c} / L_{a}=-\left(L_{c} / L_{s}+3\right) / 2+ \\
+\sqrt{\left[\left(L_{c} / L_{s}+3\right) / 2\right]^{2}-1-2 L_{c} / L_{s}+E_{\text {Л }} \sin \alpha t_{\text {И }} / 2 I_{\text {уд }} L_{s}} .
\end{gathered}
$$

Сравнение (5) и (6) показывает, что увеличение $n$ не приводит к увеличению переменных, однако расчетные соотношения значительно усложняются. Поэтому можно предположить, что при $n \gg 1$ определение индуктивности разделительных реакторов требует решения алгебраических уравнений высокого порядка или разработки методики приближенного расчета. 
Проиллюстрируем положение примером, заимствованным из [2]. Пусть $E_{\text {Л }}=1,045 \cdot 230=240 \mathrm{~B}, \alpha=20^{\circ}, L_{s}=10^{-4} \Gamma_{\mathrm{H}}, t_{\text {и }}=10^{-4}$ сек, $I_{\text {уд }}=0,5 \mathrm{~A}, L_{\mathrm{c}} / L_{s}=0,1$. Для двухпостовой СЭП $n=2$

$$
L_{c} / L_{a}=\frac{240 \cdot \sin 20^{\circ} \cdot 10^{-4}}{2 \cdot 1 \cdot 10^{-4}}-1-0,1=40 ;
$$

откуда $L_{\alpha}=0,25$ мкГн.

Для трехпостовой СЭП $n=3$

$$
L_{c} / L_{a}=-\frac{0,1+3}{2}+\sqrt{\left(\frac{0,1+3}{2}\right)^{2}-1-2 \cdot 0,1+\frac{240 \cdot \sin 20^{\circ} \cdot 10^{-4}}{2 \cdot 1 \cdot 10^{-4}}}=4,95,
$$

откуда $L_{\alpha}=2,02 \cdot$ мкГн.

Приведенный пример показывает, что с увеличением $n$ необходимая индуктивность разделительных реакторов растет быстро. Это заставляет относиться с осторожностью к надежде реализовать многопостовые $n \gg 1$ СЭП с магистральной распределительной сетью и короткими управляющими импульсами.

\section{ЛИТЕРАТ УРА}

1. Тамкиви П., Томсон Т. Изв. АН ЭССР. Физ. Матем., 31, № 4, 454-457 (1982).

2. Томсон T. Изв. АН ЭССР. Физ. Матем., 32, № 3, 311-319 (1983).

3. Ривкин T. А. Электротехника, № 3, 22-24 (1967).
Институт термофизики и электрофизики Академии наук Эстонской ССР
Поступила в редакцию 2/IV 1985

